\title{
VARIATIONAL PRINCIPLE IN THE ALGEBRA OF ASYMPTOTIC FIELDS
}

\author{
O.W. Greenberg \\ Center for Theoretical Physics \\ Department of Physics \\ University of Maryland \\ College Park, MD 20742-4111
}

\begin{abstract}
This paper proposes a variational principle for the solutions of quantum field theories in which the "trial functions" are chosen from the algebra of asymptotic fields, and illustrates this variational principle in simple cases.
\end{abstract}

\section{Introduction}

The most-used methods to find approximate solutions of quantum field theories are based on path integrals 1 1, 2, 3, 4, 5]. They have many advantages; however the Hilbert space and particle structure of field theory are not evident from this point of view. Fock space methods, such as the Tamm-Dancoff approximation [6, 7] and the discretized light cone quantization approximation (DLCQ) [8 place the Hilbert space and particle structure of the theory in the forefront; however the covariance of the theory is not evident. The functional Schrödinger picture allows intuitive guesses about the form of the solutions of a field theory to be incorporated, but this method also fails to be explicitly covariant.

Another method, less developed than those just mentioned, is the expansion in normal-ordered asymptotic fields, the "Haag expansion" or "N-quantum approximation," which applies directly only to theories without zero-mass particles. In

\footnotetext{
${ }^{1}$ Supported in part by a Semester Research Grant from the General Research Board of the University of Maryland and by the National Science Foundation; email address, owgreen@physics.umd.edu.
} 
particular, it does not apply directly to gauge theories. Nonetheless, this method can be extended to gauge theories; indeed it has been applied to quantum electrodynamics. Using the Haag expansion, one works in the algebra of asymptotic fields, and can keep creation and annihilation parts of operators on the same footing, since one can choose not to apply elements of the algebra to the vacuum, where terms with annihilation operators would annihilate the vacuum and be lost. By contrast, in the Tamm-Dancoff approximation, the annihilation parts destroy the vacuum and disappear from the calculation. In addition to losing explicit Lorentz invariance, this asymmetric treatment of the annihilation and creation parts of the fields destroys crossing symmetry. The DLCQ method also treats annihilation and creation operators asymmetrically; in addition, it suffers from a lack of explicit covariance. This complicates renormalization considerably.

Just as variational methods have been used in other approaches to quantum field theory, this paper proposes a variational principle based on the Haag expansion. Variational principles in quantum mechanics are powerful ways to go beyond perturbation theory. In quantum mechanics, the solutions lie in a Hilbert space of functions and the trial functions are chosen from this space. For example, to approximate the ground state, one can choose a wave function $\psi$ that depends on some parameters and determine the parameters by minimizing the ground state energy [9]. The exact ground state energy will be less than the approximate energy at the minimum. Many attempts have been made to carry over this approach to quantum field theories. In field theory, the ground state wave function is replaced by a vacuum wave functional that can depend on functions as well as parameters. Minimizing the vacuum energy determines the functions and parameters and yields approximate information about the solution of the theory [10, 11, 12, 13]. This approach is usually restricted to wave functionals closely related to Gaussians, because the necessary path integrals can be done only in that case. Also, this approach does not take advantage of the particle spectrum that we expect to occur in quantum field theories. For a relativistic theory, for example, the spectrum should consist of a vacuum of energy-momentum zero, one or more single-particle states (including possible bound states) with various masses (here, as in the rest of this paper, the analysis is restricted to cases without massless states), and many-particle states whose energies and momenta correspond to several massive particles. This particle structure can 
be put into a variational calculation at the outset by choosing to approximate the fields, rather than the states, and by using an expansion in asymptotic fields for the interacting fields. (Alternatively, as given below, one can use generalized free fields in the expansion of the interacting fields and derive the fact that the fields in the expansion are ordinary free fields.) Thus, in quantum field theory, assuming completeness and irreducibility of the algebra of asymptotic fields, one finds that the solutions lie in this algebra and the trial wave functions are replaced by trial operators chosen from this algebra. Rudolf Haag [14] introduced the idea to use an element in the algebra of asymptotic fields to represent the interacting field. It is fitting to call such a representation a "Haag expansion." Some applications of Haag expansions are given in [15]. While a systematic approximation using terms with normal-ordered products having arbitrarily high degree in asymptotic fields leads to amplitudes with arbitrarily many momenta and becomes intractable, a variational trial operator in the algebra of asymptotic fields can have infinite degree, but still can be parametrized in a tractable way. Section 2 formulates the variational principle and proves that the minimum picks out the solution of the field theory. Section 3 illustrate the principle with the simplest cases: a free neutral scalar field of mass $m$ with the trial operator chosen to be a generalized free field, and a free Dirac field where the trial operator is a free Dirac field. To compare with the variational method using a vacuum functional, Sec. 4 studies the $\phi^{4}$ model in $1+1$ and compares the results in the lowest approximation with the results of [10]. To show how the principle works in cases where the trial element has infinite degree, Sec. 5 applies the principle to the gradient coupling model, which has a "nucleon" Dirac field coupled to a scalar "meson" field. In that case, one can choose the trial operator for the nucleon field to be the product of an arbitrary function of a free scalar field and a free nucleon field all at a single spacetime point and the trial operator for the meson field to be a free scalar field. Section concludes with the outlook for future work.

\section{The variational principle}

Using reasonable assumptions (in particular that no massless fields are present) and a physicist's level of mathematical rigor, one can show [16] that the asymptotic fields obey the same Poincaré transformation law as the interacting fields. For example, 
for a scalar field,

$$
U(a, \Lambda) \phi^{a s}(x) U(a, \Lambda)^{\dagger}=\phi^{a s}(\Lambda x+a),
$$

$\Lambda \in S O(1,3), a \in R^{4}$. For the general case,

$$
U(a, A) \psi_{i}^{a s}(x) U(a, A)^{\dagger}=\mathcal{D}_{i j}\left(A^{-1}\right) \psi_{j}^{a s}(\Lambda x+a),
$$

where $A$ in $S L(2, C)$ is replaced by $\Lambda$ in $S O(3,1)$ for integer spin fields. One can also show that the asymptotic fields obey free field commutation relations, for example, for a scalar field,

$$
\left[\phi^{a s}(x), \phi^{a s}(y)\right]_{-}=i \Delta\left(x-y ; m^{2}\right),
$$

where

$$
i \Delta\left(x, m^{2}\right)=(2 \pi)^{-3} \int d^{4} k \epsilon\left(k^{0}\right) \delta\left(k^{2}-m^{2}\right) \exp (-i k \cdot x)
$$

is the Pauli-Jordan commutator function. These two conditions, together with the requirement that the vacuum have zero energy, imply that the generators of the Poincaré group are the free bilinear functionals of the asymptotic fields; in particular that (apart from a constant which is the vacuum matrix element of the Hamiltonian) the Hamiltonian is diagonalized by the asymptotic fields,

$$
P^{0} \equiv H=\text { const. }+\sum_{i} H_{\text {free }}\left[\phi_{i}^{a s}\right] .
$$

To see this, assume $H$ has an arbitrary expansion in asymptotic fields,

$$
\begin{aligned}
H= & F^{(0)}+\sum_{j=1}^{n} F_{-j}^{(1)} A_{j}^{i n}(\mathbf{0})+\sum_{j=1}^{n} F_{+}^{(1)} A_{j}^{i n}(\mathbf{0})^{\dagger} \\
& +\sum_{s, t}\left[\int \frac { d ^ { 3 } k } { 2 \omega _ { k } } \left[F_{--}^{(2)} s, t(\mathbf{k}) A_{s}^{i n}(\mathbf{k}) A_{t}^{i n}(-\mathbf{k}) \exp \left(-2 i \omega_{k} x^{0}\right)+F_{+-s, t}^{(2)}(\mathbf{k}) A_{s}^{i n}(\mathbf{k})^{\dagger} A_{t}^{i n}(\mathbf{k})\right.\right. \\
& \left.+F_{++}^{(2)} s(\mathbf{k}) A_{s}^{i n}(\mathbf{k})^{\dagger} A_{t}^{i n}(-\mathbf{k})^{\dagger} \exp \left(2 i \omega_{k} x^{0}\right)\right]+\sum_{n>2}^{\infty} \int \frac{d^{3 n} k}{\prod \omega_{k_{i}}} F^{(n)}\left(k_{i}\right) \\
& \times: \prod_{j} A_{j}^{i n}\left(\mathbf{k}_{i}\right)^{()}: \delta\left(\sum \pm \mathbf{k}_{i}\right) \exp \left(i \pm \omega_{k_{i}} t \pm \mathbf{k}_{i} \cdot \mathbf{x}_{i}\right), \quad \omega_{k}=\sqrt{\mathbf{k}^{2}+m^{2}}
\end{aligned}
$$

$A^{\text {in }}\left(\mathbf{k}_{i}\right)^{()}$stands for either the creation or the annihilation operator, normalized relativistically. From Eq.(3) and the Fourier transform

$$
\phi^{i n}(x)=(2 \pi)^{-3 / 2} \int d^{4} k \tilde{\phi}^{i n}(k) \delta_{m}(k) \exp (-i k \cdot x)
$$




$$
\phi^{(i n)}(k) \delta_{m}(k)=\theta\left(k^{0}\right) \frac{A^{i n}(\mathbf{k})}{2 \omega_{k}} \delta\left(k^{0}-\omega_{k}\right)+\theta\left(-k^{0}\right) \frac{A^{i n}(-\mathbf{k})^{\dagger}}{2 \omega_{k}} \delta\left(k^{0}+\omega_{k}\right),
$$

the commutation relations of the creation and annihilation operators are

$$
\left[A^{i n}(\mathbf{k}), A^{i n \dagger}(\mathbf{l})\right]_{-}=2 \sqrt{\mathbf{k}^{2}+m^{2}} \delta(\mathbf{k}-\mathbf{l})
$$

The nonrelativistically normalized annihilation and creation operators are $a^{(i n)}\left(\mathbf{k}_{i}\right)^{()}=$ $\left(2 E_{k}\right)^{-1 / 2} A^{i n}\left(\mathbf{k}_{i}\right)^{()}, E_{k}=k^{0}>0$. Put the form for $H$ into the infinitesimal form of Eq.(11),

$$
i\left[H, \phi^{a s}(x)\right]_{=} \partial_{0} \phi^{a s}(x) .
$$

The commutation relation, Eq.(3 or 2), say that a normal-ordered term in $H$ with $n$ factors of $\phi^{a s}$ will contribute to a term with $n-1$ factors of $\phi^{a s}$ on the right-handside of Eq.(10). Since the right-hand-side of Eq.(10) is linear in $\phi^{a s}$, the only terms allowed in $H$ are those with $n=0$ or $n=2$. The $n=0$ term is the vacuum energy, and because $\phi^{a s}$ is a free field, the $n=2$ term is the free field Hamiltonian.

The Haag expansion of the interacting fields, stated for simplicity for a single neutral scalar field with only scalar bound states, is

$$
\phi(x)=\sum_{n=0}^{\infty} \frac{1}{n !} \sum_{j_{i}} \int \sum_{j} d^{4 n} x_{i} f^{(n)}\left(\left\{x-x_{i}\right\}\right): \prod_{i=1}^{n} \phi_{j_{i}}^{i n}\left(x_{i}\right):
$$

where the $\phi_{j_{i}}^{\text {in }}$ include in fields for stable bound states, if there are any. The term $f^{(0)}=v$ is a constant that occurs for a scalar field when symmetry is broken. The term with $f^{(1)}=1$ is just the in field with coefficient one to fix field strength renormalization. In momentum space, the expansion is

$$
\begin{aligned}
\tilde{\phi}(k)= & (2 \pi)^{3 / 2} v \delta(k)+\tilde{\phi}^{i n}(k) \delta_{m}(k)+\sum_{n=2}^{\infty} \frac{1}{n !} \sum_{j} \int \tilde{f}^{(n)}\left(k_{1}, \ldots, k_{n}\right) \\
& \times: \prod_{i=1}^{n} \tilde{\phi}_{j}^{i n}\left(k_{i}\right) \delta_{m_{j}}\left(k_{i}\right): \delta\left(k-\sum k_{i}\right) \prod_{i=1}^{n} d^{4} k_{i},
\end{aligned}
$$

$f^{(n)}\left(x_{1}, \ldots, x_{n}\right)=(2 \pi)^{-3 / 2-5 n / 2} \int \tilde{f}^{(n)}\left(k_{1}, \ldots, k_{n}\right) \exp \left(-i \sum k_{i} \cdot x_{i}\right) \prod d^{4} k_{i}$. Hermiticity of the field implies $\tilde{f}^{(n)}\left(k_{1}, \ldots, k_{n}\right)=\tilde{f}^{(n) *}\left(-k_{1}, \ldots,-k_{n}\right)$. Similar expansions hold for 
out fields. When this expansion is inserted into the Hamiltonian, the result is an infinite series of the form already given in Eq.(6).

For a given Haag expansion, Eq.(12), parametrized by $v$ and the $\tilde{f}^{(n)}$ 's, the $F^{(n)}$ 's of Eq.(6) are functionals of $v$ and the $\tilde{f}^{(n)}$ 's. From the discussion above, for the exact solution $F^{(n)}=0$ for $n=1$ and for all $n>2, F^{(2)}=0$ for the -- and ++ cases, and $F_{+-}^{(2)}$ is minimum. In other words, as just discussed, the Hamiltonian is the sum of free-field Hamiltonians for each in field, together with a constant term which is the vacuum matrix element of $H$. The condition that the Haag expansion diagonalizes $H$ leads to an infinite set of nonlinear integral equations in $v$ and the $\tilde{f}^{(n)}$ 's. A solution to this set of equations is equivalent to the solution of the field theory in all sectors. In practice, it will be difficult to find an exact solution. Variational methods can yield approximate solutions. Many different conditions can be imposed on the $F^{(n)}$ 's to find an approximation solution from a variational principle. One such condition is to minimize the sum $\mathcal{Q}$ (with possible weighting factors $\lambda_{n}$ ) of the integral of the absolute squares of the coefficients of the operator terms,

$$
\begin{aligned}
\mathcal{Q}= & \lambda_{0}\left|F^{(0)}\right|^{2}+\lambda_{-}\left|F_{-}^{(1)}\right|^{2}+\lambda_{+}\left|F_{+}^{(1)}\right|^{2} \\
& +\lambda_{--} \int d^{3} k\left|F_{--}^{(2)}(k,-k)\right|^{2}+\lambda_{+-} \int d^{3} k\left|F_{+-}^{(2)}(k, k)\right|^{2} \\
& +\lambda_{++} \int d^{3} k\left|F_{+-}^{(2)}(k,-k)\right|^{2}+\sum_{n} \lambda_{n} \int \prod d^{3} k_{i}\left|F^{(n)}\left(k_{1}, \ldots, k_{n}\right)\right|^{2}
\end{aligned}
$$

(here, to simplify the notation, I dropped the subscripts that label the possibly different asymptotic fields). The $\lambda$ 's are arbitrary positive or vanishing numbers that can be chosen to control the weight attached to each term. If the Haag expansion of the interacting field has finite degree, the terms in the Hamiltonian of highest degrees in in-fields cannot possibly vanish, so the $\lambda$ 's for such terms should be chosen to vanish. For infinite degree Haag expansions which remove the restriction to weak coupling one can keep all the $\lambda$ 's positive. For the exact solution all offdiagonal terms vanish and the diagonal terms are minimum, so this principle gives the exact solution of the theory in all sectors at the absolute minimum if all the $\lambda$ 's are positive. If all $\lambda^{\prime}$ s except the one multiplying the $a^{\dagger} a$ term for a bound state are chosen to vanish, this variational principle reduces to the quantum mechanical one 
for the bound state. Another possible condition is to minimize the integral of the absolute square of the coefficients,

$$
\int \prod d^{3} k_{i}\left|F^{(n)}\left(k_{1}, \ldots, k_{n}\right)\right|^{2}
$$

for some set of values of $n$ and for each combination of creation and annihilation parts of the operators. One can also choose to require that some set of sums of the positive terms in Eq.(13) vanish. Each of these possibilities gives a set of equations for the solution of the variational principle. The specific conditions one should impose in a given problem should be chosen by experience.

\section{Application to the free field}

The simplest case on which to test this variational principle is the free field. The Hamiltonian for a free neutral scalar field is

$$
H=\frac{1}{2} \int d^{3} x\left(\dot{\phi}^{2}+(\nabla \phi)^{2}+m^{2} \phi^{2}\right)
$$

As a trial operator, choose a generalized free field, $\phi_{g f f}$, whose two-point function has an unknown positive measure $\rho\left(\kappa^{2}\right)$. To avoid the trivial case where $\rho=0$, require $\int_{0}^{\infty} \rho\left(\kappa^{2}\right) d \kappa^{2}$ to have a fixed positive value; the exact value doesn't matter. Let the field $\phi_{g f f}$ be represented as the sum of a term, $\phi_{d i s}\left(x ; \mu^{2}\right)$, with a discrete weight at mass $\mu^{2}$ and a term with a continuous weight $\sigma, \phi_{c o n}(x ; \sigma)$,

$$
\begin{gathered}
\phi_{g f f}(x)=\phi_{\text {dis }}\left(x ; \mu^{2}\right)+\phi_{\text {con }}(x ; \sigma), \\
\phi_{\text {dis }}\left(x ; \mu^{2}\right)=\frac{1}{(2 \pi)^{3 / 2}} \int \frac{d^{3} k}{2 E_{\mu}(\mathbf{k})}\left[A(\mathbf{k}) e^{-i k \cdot x}+A^{\dagger}(\mathbf{k}) e^{i k \cdot x}\right], \quad k^{0}=E_{\mu}(\mathbf{k}), \\
\phi_{c o n}(x ; \sigma)=\frac{1}{(2 \pi)^{3 / 2}} \int d \kappa^{2} \frac{d^{3} k}{2 E_{\kappa}(\mathbf{k})}\left[B\left(\mathbf{k} ; \kappa^{2}\right) e^{-i k \cdot x}+B^{\dagger}\left(\mathbf{k} ; \kappa^{2}\right) e^{i k \cdot x}\right], \\
k^{0}=E_{\kappa}(\mathbf{k})=\sqrt{\mathbf{k}^{2}+\kappa^{2}} .
\end{gathered}
$$

The relativistically normalized commutation relations are

$$
\left[A(\mathbf{k}), A^{\dagger}(\ell)\right]_{-}=2 E_{\mu}(\mathbf{k}) \delta(\mathbf{k}-\ell)
$$




$$
\left[B\left(\mathbf{k} ; \kappa^{2}\right), B^{\dagger}\left(\ell ; \lambda^{2}\right)\right]_{-}=2 E_{\kappa}(\mathbf{k}) \delta(\mathbf{k}-\ell) \delta\left(\kappa^{2}-\lambda^{2}\right) \sigma\left(\kappa^{2}\right),
$$

other commutators vanish. To find the minimum of the energy of a given particle, one should minimize the coefficient of the $a^{\dagger} a$ term. The relations between the relativistically (capital letters) and nonrelativistically (lower-case letters) normalized operators are

$$
\begin{aligned}
A(\mathbf{k}) & =\sqrt{2 E_{\mu}(\mathbf{k})} a(\mathbf{k}), \\
B\left(\mathbf{k} ; \kappa^{2}\right) & =\sqrt{2 E_{\kappa}(\mathbf{k})} b\left(\mathbf{k} ; \kappa^{2}\right) .
\end{aligned}
$$

When the trial operator $\phi_{g f f}$ is inserted into the free scalar Hamiltonian, the result is

$$
\begin{aligned}
& H=H_{d i s}+H_{d i s-c o n}+H_{c o n}, \\
& H_{d i s}=\frac{1}{2} \int \frac{d^{3} k}{2 E_{\mu}(\mathbf{k})}\left(E_{\mu}(\mathbf{k})^{2}+\mathbf{k}^{2}+m^{2}\right) \delta(\mathbf{0}) \\
& +\frac{1}{2} \int \frac{d^{3} k}{2 E_{\mu}(\mathbf{k})}\left\{\left[\left(\mathbf{k}^{2}+m^{2}-E_{\mu}(\mathbf{k})^{2}\right): a(\mathbf{k}) a(-\mathbf{k}): e^{-2 i E_{\mu}(\mathbf{k}) t}+h . c .\right]\right. \\
& \left.+2\left(E_{\mu}(\mathbf{k})^{2}+\mathbf{k}^{2}+m^{2}\right): a^{\dagger}(\mathbf{k}) a(\mathbf{k}):\right\} \\
& H_{\text {dis-con }}=\int \frac{d \lambda^{2} d^{3} k}{2 \sqrt{E_{\mu}(\mathbf{k}) E_{\lambda}(\mathbf{k})}}\left[\left(-E_{\mu}(\mathbf{k}) E_{\lambda}(\mathbf{k})+\mathbf{k}^{2}+m^{2}\right): a(\mathbf{k}) b\left(-\mathbf{k} ; \lambda^{2}\right):\right. \\
& \times e^{-i\left(E_{\mu}(\mathbf{k})+E_{\lambda}(\mathbf{k})\right) x^{0}}+\left(E_{\mu}(\mathbf{k}) E_{\lambda}(\mathbf{k})+\mathbf{k}^{2}+m^{2}\right) \\
& \times: b^{\dagger}\left(\mathbf{k} ; \lambda^{2}\right) a(\mathbf{k}): e^{-i\left(E_{\mu}(\mathbf{k})-E_{\lambda}(\mathbf{k})\right) x^{0}}+\text { h.c.] }, \\
& H_{\text {con }}=\frac{1}{2} \int \frac{d \kappa^{2} d^{3} k}{2 E_{\kappa} \mathbf{k}}\left(E_{\kappa}(\mathbf{k})^{2}+\kappa^{2}+m^{2}\right) \sigma\left(\kappa^{2}\right) \delta(\mathbf{0})+\frac{1}{2} \int \frac{d \kappa^{2} d \lambda^{2} d^{3} k}{2 \sqrt{E_{\kappa}(\mathbf{k}) E_{\lambda}(\mathbf{k})}} \\
& \times\left\{\left[\left(-E_{\kappa}(\mathbf{k}) E_{\lambda}(\mathbf{k})+\mathbf{k}^{2}+m^{2}\right): b\left(\mathbf{k} ; \kappa^{2}\right) b\left(-\mathbf{k} ; \lambda^{2}\right):\right.\right. \\
& \left.\times e^{-i\left(E_{\kappa}(\mathbf{k})+E_{\lambda}(\mathbf{k})\right) x^{0}}+\text { h.c. }\right]+2\left(E_{\kappa}(\mathbf{k}) E_{\lambda}(\mathbf{k})+\mathbf{k}^{2}+m^{2}\right) \\
& \left.\times: b^{\dagger}\left(\mathbf{k} ; \lambda^{2}\right) b\left(\mathbf{k} ; \kappa^{2}\right): e^{-i\left(E_{\kappa}(\mathbf{k})-E_{\lambda}(\mathbf{k})\right) x^{0}}\right\}
\end{aligned}
$$

For the terms of the form 1 (vacuum energy) or $a^{\dagger} a$ (particle energy), we must minimize the energy $\left(2 \mathbf{k}^{2}+\mu^{2}+m^{2}\right) / \sqrt{\mathbf{k}^{2}+\mu^{2}}$ with respect to $\mu^{2}$, or, alternatively, 
minimize the same energy written as $\left[E_{\mu}(\mathbf{k})^{2}+\mathbf{k}^{2}+m^{2}\right] / E_{\mu}(\mathbf{k})$ with respect to $E_{\mu}(\mathbf{k})$; for terms of the form $a a$ or $a^{\dagger} a^{\dagger}$ we minimize the absolute value squared of the coefficient (since we want to bring the Hamiltonian to diagonal form). For terms of the form $b^{\dagger} b$, we must minimize the energy,

$$
\frac{E_{\kappa}(\mathbf{k}) E_{\lambda}(\mathbf{k})+\mathbf{k}^{2}+m^{2}}{2 \sqrt{E_{\kappa}(\mathbf{k}) E_{\lambda}(\mathbf{k})}},
$$

with respect to $\kappa^{2}$ and $\lambda^{2}$. For terms of the form $b^{\dagger} a$ or $a^{\dagger} b$, similar expressions have their minima for $\sigma$ concentrated at $\kappa^{2}=\mu^{2}=m^{2}$. For terms of the forms $a a$, $a^{\dagger} a^{\dagger}, a b, a^{\dagger} b^{\dagger}, b b$, and $b^{\dagger} b^{\dagger}$, the squares of the coefficients vanish for $\mu^{2}=m^{2}$ and $\sigma$ concentrated at $\kappa^{2}=m^{2}$. For the contributions from $\phi_{c o n}$, the minimum occurs for $\sigma$ at $\kappa^{2}=m^{2}$. The net result is that the minimum of the operator $\mathrm{H}$ occurs at

$$
\phi_{g f f}(x)=\phi_{d i s}\left(x ; m^{2}\right), \phi_{c o n}=0 ;
$$

i.e., for a free field of the mass in the Lagrangian. This is no surprise. In general, for theories without massless fields or particles, we would assume free field form for the asymptotic fields without doing a calculation. (Note that these results require minimizing an expression that has dimensions of energy.)

If one assumes a free field in the corresponding calculation of the Hamiltonian for the Dirac field the $b^{\dagger} b$ and $d^{\dagger} d$ terms are diagonal in helicity; the $b d$ and $b^{\dagger} d^{\dagger}$ terms are not. The minimum of the absolute valued squared coefficients also yields the result $\mu^{2}=m^{2}$.

\section{Application to the $\phi^{4}$ model in $1+1$}

A less trivial, but still elementary, example is the $\phi^{4}$ theory in one space, one time dimension. This model was studied recently using a variational method by G. Tiktopoulos [10]. (The literature on variational calculations can be traced from [10, 11, 12, 13].) The example just below illustrates the variational principle in the algebra of asymptotic fields for this theory in the lowest non-trivial approximation, where the calculations can be done easily by hand. Higher approximations require 
symbolic manipulation programs that have been developed using Reduce 3.5. We will report on the results of higher approximations separately. The Lagrangian is

$$
\mathcal{L}=\frac{1}{2}\left(\partial_{\mu} \phi \cdot \partial^{\mu} \phi-m_{u}^{2} \phi^{2}\right)-\frac{\lambda}{4 !} \phi^{4}
$$

The Hamiltonian density is

$$
\mathcal{H}=\frac{1}{2}\left(\dot{\phi}^{2}+\phi^{\prime 2}+m_{u}^{2} \phi^{2}\right)+\frac{\lambda}{4 !} \phi^{4} .
$$

For a theory that has no massless particles or fields, the (on-shell) asymptotic fields will be an irreducible set of operators in which to expand the interacting field. The demonstration given above that the free asymptotic fields diagonalize the free Hamiltonian supports this expectation. The lowest variational ansatz is then

$$
\phi(x)=v+\phi^{(i n)}\left(x ; m^{2}\right),
$$

where the constant $v$ is a symmetry-breaking vacuum matrix element of $\phi$ that may occur due to radiatively-induced symmetry breaking and $\phi^{(i n)}$ is a free field of unknown mass $m$. Substituting this ansatz and re-normal ordering, the Hamiltonian becomes

$$
\begin{aligned}
\mathcal{H}= & {\left[\frac{1}{2}\left(<\dot{\phi}_{0}^{2}>+<\phi_{0}^{\prime 2}>\right)+m_{u}^{2}\left(v^{2}+<\phi_{0}^{2}>\right)\right.} \\
& \left.+\frac{\lambda}{4 !}\left(v^{4}+6 v^{2}<\phi_{0}^{2}>+3<\phi_{0}^{2}>^{2}\right)\right] \\
& +\left[\left(m_{u}^{2} v+\frac{\lambda}{6} v^{3}\right)+\frac{\lambda}{2} v<\phi_{0}^{2}>\right]: \phi_{0}: \\
& +\frac{1}{2}\left\{: \dot{\phi}_{0}^{2}+: \phi_{0}^{\prime 2}:+\left[m_{u}^{2}+\frac{\lambda}{2}\left(v^{2}+<\phi_{0}^{2}>\right)\right]: \phi_{0}^{2}:\right\} \\
& +\frac{\lambda}{6} v: \phi_{0}^{3}:+\frac{\lambda}{24}: \phi_{0}^{4}:,
\end{aligned}
$$

where $\phi_{0}$ stands for $\phi^{(i n)}\left(0 ; m^{2}\right)$. The vacuum matrix element is just the first square bracket above. The coefficient of the term in $(1 / 2): \phi_{0}^{2}:$ should be the square of the physical mass $m_{v}^{2}$, i.e.

$$
m_{v}^{2}=m_{u}^{2}+\frac{\lambda}{2}\left(v^{2}+<\phi_{0}^{2}>\right) .
$$


This is the gap equation in this approximation. Following Tiktopoulos, one can verify this below by minimizing the vacuum energy density which is a functional of the energy, $E(k)$, of the trial in-field quanta of momenta $k$ with respect to this energy. Regulate the vacuum and other divergent expressions with a momentumspace cut-off; the two-point function is then

$$
\left\langle\phi_{0}(x) \phi_{0}(y)\right\rangle=\frac{1}{2 \pi} \int_{-\Lambda}^{\Lambda} \frac{d k}{2 E(k)} e^{-i E(k) x^{0}+k x^{1}} .
$$

Then

$$
\left\langle\dot{\phi}_{0}^{2}\right\rangle=\frac{1}{4 \pi} \int_{-\Lambda}^{\Lambda} d k E(k), \quad\left\langle\phi_{0}^{\prime 2}\right\rangle=\frac{1}{4 \pi} \int_{-\Lambda}^{\Lambda} d k \frac{k^{2}}{E(k)},\left\langle\phi_{0}^{2}\right\rangle=\frac{1}{4 \pi} \int_{-\Lambda}^{\Lambda} d k \frac{d k}{E(k)} .
$$

The vacuum energy density is

$$
\begin{aligned}
<\mathcal{H}>= & \frac{1}{8 \pi} \int_{-\Lambda}^{\Lambda} \frac{d k}{E(k)}\left(E(k)^{2}+k^{2}+m_{v}^{2}\right)+\frac{1}{2} m_{u}^{2} v^{2}+\frac{\lambda}{24} v^{4} \\
& +\frac{\lambda}{16 \pi} v^{2} \int_{-\Lambda}^{\Lambda} \frac{d k}{E(k)}+\frac{\lambda}{2^{7} \pi^{2}}\left(\int_{-\Lambda}^{\Lambda} \frac{d k}{E(k)}\right)^{2} .
\end{aligned}
$$

The minimum is given by

$$
\begin{aligned}
\frac{\delta\langle H\rangle}{\delta E(k)} & =\frac{1}{8 \pi}\left(1-\frac{k^{2}+m_{u}^{2}}{E(k)^{2}}\right)-\frac{\lambda}{16 \pi} \frac{v^{2}}{E(k)^{2}}-\frac{\lambda}{2^{7} \pi^{2}} \int_{-\Lambda}^{\Lambda} \frac{d k^{\prime}}{E\left(k^{\prime}\right)} \frac{1}{E(k)^{2}} \\
& =0 .
\end{aligned}
$$

After multiplying by $8 \pi E(k)^{2}$, the result is the gap equation, so $m^{2}=m_{v}^{2}$ as given by $(33)$.

Since the gap equation relates the physical mass $m_{v}^{2}$, which should be finite, to the bare mass and the divergent integrals cut-off at $\Lambda$, one can follow Tiktopoulos in introducing another finite mass, $M$, via

$$
M^{2}=m_{u}^{2}+\frac{\lambda}{8 \pi} \int_{-\Lambda}^{\Lambda} \frac{d k}{E(k)} .
$$

This form of renormalization can replace the usual one, since here only the mass is renormalized. As Tiktopoulos showed, the gap equation then relates finite quantities,

$$
m_{v}^{2}=M^{2}+\frac{\lambda}{2} v^{2}+\frac{\lambda}{8 \pi} \ln \frac{M^{2}}{m_{v}^{2}} .
$$


The minimum of the vacuum energy density is

$$
\begin{aligned}
\langle\mathcal{H}\rangle= & \frac{1}{4 \pi} \int_{-\Lambda}^{\Lambda} d k|k|-\frac{1}{2 \lambda}\left(M^{2}-\frac{\lambda}{8 \pi} \int_{-\Lambda}^{\Lambda} \frac{d k}{\sqrt{k^{2}+m^{2}}}\right)^{2}+\frac{1}{8 \pi} m_{v}^{2} \\
& +\frac{\lambda}{24} v^{4}+\frac{1}{2} v^{2}\left(M^{2}+\frac{\lambda}{8 \pi} \ln \frac{M^{2}}{m_{v}^{2}}\right)+\frac{1}{2 \lambda}\left(M^{2}+\frac{\lambda}{8 \pi} \ln \frac{M^{2}}{m_{v}^{2}}\right)^{2}
\end{aligned}
$$

again in agreement with Tiktopoulos. Thus the present variational calculation leads to the same results as does that of Tiktopoulos in the lowest approximation. This agreement will not persist in higher approximations. Tiktopoulos adds Gaussians to his vacuum functional in order to improve his approximation. The Haag expansion suggests a different form of the higher-order terms, with higher-degree normalordered products of asymptotic fields, in the expansion of the interacting field. These are very different approximations. If the in (or out) fields are irreducible, then the Haag expansion will approximate the exact solution in theories without massless fields. In higher approximations, the present variational method will place emphasis on minimizing the absolute square of the coefficients of the non-quadratic terms in $\mathcal{H}$ and on minimizing the coefficients of the $a^{\dagger} a$ terms which correspond to the energies of the asymptotic quanta.

\section{Application to the derivative coupling model in $1+3$}

To illustrate a trial function that is of infinite degree, consider the derivative coupling model, with

$$
\mathcal{L}=Z_{2} \bar{\psi}(i \not \partial+g \not \partial \phi-M) \psi+\frac{1}{2}\left(\partial_{\mu} \phi \cdot \partial^{\mu} \phi-m^{2} \phi^{2}\right),
$$

where $\phi$ and $\psi$ are renormalized fields. The Hamiltonian is

$$
H=\int d^{3} x\left[Z_{2} \bar{\psi}\left(i \gamma^{j} \partial^{j}+g \gamma^{j} \partial^{j} \phi+M\right) \psi+\frac{1}{2}\left(\dot{\phi}^{2}+\left(\partial^{j} \phi\right)^{2}+m^{2} \phi^{2}\right)\right]
$$

Assume $\phi=\phi_{0}, \psi=: f\left(\phi_{0}\right): \psi_{0}$. The Hamiltonian becomes

$$
\begin{aligned}
H= & \int d^{3} x\left[Z_{2} \bar{\psi}_{0}: f\left(\phi_{0}\right)^{\dagger}:\left(i: f^{\prime}\left(\phi_{0}\right) \gamma^{j} \partial^{j} \phi_{0}:+g \gamma^{j} \partial^{j} \phi_{0}: f\left(\phi_{0}\right):+i: f\left(\phi_{0}\right): \gamma^{j} \partial^{j}+\right.\right. \\
& \left.\left.+M: f\left(\phi_{0}\right):\right) \psi_{o}+\frac{1}{2}\left(\dot{\phi}_{0}{ }^{2}+\left(\partial^{j} \phi_{0}\right)^{2}+m^{2} \phi_{0}^{2}\right)\right] .
\end{aligned}
$$


The first two terms in the bracket will cancel if

$$
i f^{\prime}+g f=0
$$

where the fact that $\left\langle\partial^{j} \phi \phi\right\rangle=0$ allows removing $\partial^{j} \phi_{0}$ from the normal-ordered product in the first term. Field strength renormalization of $\psi$ requires $f(0)=1$. The solution of this is $f(x)=\exp (i g x)$. Evaluation of : $f\left(\phi_{0}\right)^{\dagger}:: f\left(\phi_{0}\right)$ : using the Baker-Hausdorff-Campbell theorem gives $\exp \left(g^{2}\langle\phi(x) \phi(y)\rangle\right)$ in the limit $x \rightarrow y$ for this product. This determines

$$
\frac{1}{Z_{2}}=\lim _{x \rightarrow y} \exp \left(g^{2}\langle\phi(x) \phi(y)\rangle\right)
$$

Thus the solution that reduces the Hamiltonian to free field form is

$$
\psi(x)=: \exp \left(i g \phi_{0}(x)\right): \psi_{0}(x), \quad \phi(x)=\phi_{0}(x)
$$

(Antisymmetrization of the Fermi fields $\psi$ and $\psi^{\dagger}$ above complicates the calculation, but does not change the outcome.)

\section{Outlook for future work}

Much must be done to make this idea into a useful tool for field theory calculations. The method should be applied to examples with a finite number of terms in the Haag expansion that serves as the trial element. Calculations of vertex functions in an all-scalar relativistic model with the interaction $\phi^{2} \chi$ are ongoing. This method will be used to calculate hydrogen bound states with the soft photon cloud taken into account for the charged particles. The full power of the method will become apparent only when infinite degree Haag expansions, parametrized in a tractable way, are used. The derivative coupling model discussed above is a trivial example of such a parametrization. As mentioned above, the soft photon cloud around a charged particle will be represented using asymptotic fields in a later paper. Realistic models will be much more difficult to treat. We must also confront the problem of the coupling of high-energy and low-energy modes pointed out by Feynman[17]. The step to nonabelian gauge theories in which the confined fields do not have asymptotic fields will be the most difficult step. Hopefully the case of electrodynamics, where 
the charged fields acquire a cloud of soft photons, which we believe we know how to treat using asymptotic fields, will serve as a stepping stone to the nonabelian case.

\section{Acknowledgements}

I am happy to thank Manoj Banerjee, Zacharia Chacko, Vigdor Teplitz, and Ching-Hung Woo for helpful discussions. I am greatly indebted to George Tiktopoulos for extensive clarifications of his paper, as well as for helpful comments about a draft of this paper. I thank Shmuel Nussinov, Daniel Phillips, and Joseph Sucher for useful suggestions about an earlier version of this article. It is a pleasure to thank Yasuo Umino for many discussions about this work, for collaboration in developing Reduce codes, as well as joint work on extensions to other problems.

\section{References}

[1] M.E. Peskin and D.V. Schroeder, An Introduction to Quantum Field Theory (Addison-Wesley, Reading, 1995).

[2] S. Weinberg, The Quantum Theory of Fields, Vol. I, Foundations; Vol. II, Modern Applications (Cambridge, Cambridge, 1995, 1996).

[3] R.P. Feynman, Rev. Mod. Phys. 20, 367 (1948); R.P. Feynman and A.R. Hibbs, Quantum Mechanics and Path Integrals, (McGraw Hill, New York, 1965).

[4] K. Wilson, Phys. Rev. D 10, 2445 (1974).

[5] M. Creutz, Quarks, Gluons and Lattices, (Cambridge, Cambridge, 1983).

[6] I.E. Tamm, J. Phys (USSR) 9, 449 (1945).

[7] S.M. Dancoff, Phys. Rev. 78, 382 (1950).

[8] S.J. Brodsky, H.-C. Pauli, and S.S. Pinsky, "Quantum Chromodynamics and Other Fields Theories on the Light Cone," hep-ph/9705477.

[9] E. Merzbacher, Quantum Mechanics, (Wiley, New York, 1961).

[10] G. Tiktopoulos, "Variational Wave Functionals in Quantum Field Theory," hep-th/9705230. 
[11] J.H. Yee, "Variational Approach to Quantum Field Theory: Gaussian Approximation and the Perturbative Expansion around It," hep-th/9707234.

[12] W.E. Brown and I.I. Kogan, "A Variational Approach to the QCD Wavefunctional: Calculation of the QCD $\beta$-Function," hep-th/9705136.

[13] I.I. Kogan and A. Kovner, Phys. Rev. D 51, 1948 (1995) and Phys. Rev. D 52, 3719 (1995).

[14] R. Haag, K. Dan Vidensk. Selsk. Mat-Fys. Medd. 29,(12) (1955).

[15] O.W. Greenberg, Phys. Rev. B 139,1038 (1965); erratum, Phys. Rev. 156, 1742 (1967); O.W. Greenberg and R. Genolio, Phys. Rev., 150, 1070 (1966); A. Raychaudhuri, Phys. Rev. D 18, 4658 (1978), and University of Maryland thesis (1977); O.W. Greenberg, S. Nussinov and J. Sucher, Phys. Lett. 70B, 465 (1977); O.W. Greenberg, Prog. Theor. Phys. Supp. 86, 60 (1986); Phys. Rev. D 47, 331 (1993); O.W. Greenberg and P. K. Mohapatra, Phys. Rev. D 34, 1136 (1986); O.W. Greenberg and L. Orr, Phys. Rev. D 36, 1240 (1987); O.W. Greenberg, R. Ray and F. Schlumpf, Phys. Lett. B 353, 284 (1995). Related work appears in F. Gross, Phys. Rev. 186, 1448 (1969); K. Johnson, Phys. Rev. D 4, 1101 (1972); M. Bander, Phys. Rev. Lett. 47, 549 (1981); 47, 1419E (1981).

[16] O.W. Greenberg, The Asymptotic Condition in Quantum Field Theory, Princeton Univ. PhD Thesis, 1956.

[17] R.P. Feynman, in Proc. Int. Workshop on Variational Calculations in Quantum Field Theory, (World Scientific, Singapore, 1988), ed. L. Polley and D.E.L. Pottinger, p 28. 